\title{
La ausencia de la familia en el proceso de aprendizaje de la comprensión lectora: la experiencia en tres grupos del nivel de I y II ciclo de la enseñanza general básica
}

The absence of the family in the learning process of reading comprehension: the experience in three groups Primary Education

\section{Volumen 20, Número 1}

Enero - Abril

pp. 1-24

María Nidia González Araya

Helvetia Cárdenas Leitón

\section{Citar este documento según modelo APA}

González Araya, María Nidia y Cárdenas Leitón, Helvetia. (2020). La ausencia de la familia en el proceso de aprendizaje de la comprensión lectora: la experiencia en tres grupos del nivel de I y II ciclo de la enseñanza general básica. Revista Actualidades Investigativas en Educación, 20(1), 1-24. Doi. 10.15517/aie.v20i1.40158 


\title{
La ausencia de la familia en el proceso de aprendizaje de la comprensión lectora: la experiencia en tres grupos del nivel de I y II ciclo de la enseñanza general básica
}

The absence of the family in the learning process of reading comprehension: the experience in three groups Primary Education

\author{
María Nidia González Araya ${ }^{1}$ \\ Helvetia Cárdenas Leitón ${ }^{2}$
}

\begin{abstract}
Resumen: Este artículo es producto de una investigación cualitativa realizada entre los años 2017-2018 que estudió la injerencia que tiene el uso de las TIC y otros mediadores culturales, entre ellos la familia en el proceso de la comprensión lectora. Para el caso particular del presente artículo, el objetivo fue reconocer el nivel de participación de la familia en la promoción de la lectura de sus hijos e hijas, considerando, a la familia como un mediador cultural. Colaboraron ocho docentes de tres centros educativos del nivel de Educación Primaria, dos de la Dirección Regional de Educación de Alajuela y una de la Dirección Regional de San Ramón, Costa Rica y sus respectivos grupos de cuarto y sexto año y asimismo, setenta y ocho padres y madres de familia de estos alumnos y alumnas. Como principales técnicas de recolección de datos, se utilizaron: el cuestionario, la entrevista y la observación participante. Entre los resultados más destacados, se obtuvo que el involucramiento familiar en el proceso de aprendizaje de las habilidades lingüísticas y específicamente de la comprensión lectora, es escaso o nulo en algunos casos, lo cual deviene en un proceso donde se involucra únicamente el o la docente y representa un ínfimo desarrollo de destrezas lectoras por parte de las niñas y los niños en el ámbito familiar y por ende, en la poca comprensión de los textos y el bajo rendimiento académico.
\end{abstract}

Palabras clave: proceso de aprendizaje, comprensión lectora, educación primaria, familia.

\begin{abstract}
The present article is the product of a qualitative study about the influence of TIC and other cultural mediators in the process of reading comprehension at the great school level. Despite the fact that this study purported to attain a series of objectives, in the specific case of the present article, only one of them is developed. Such and objective aimed to recognize the level of participation of the family in the promotion of reading in their children. Participants were ten teachers from three educational institutions at the great school level and six fourth and sixth level groups from educational institutions from two Regional School Districts from the West and Alajuela. Likewise, forty-eight parents of these children participated. The main data collection techniques used included questionnaires for the families, teacher interviews and participant observations to the groups. In accordance with the afore mentioned objective, results were that family involvement in the learning of linguistic abilities such as reading is scares or none in some cases. This is evidence to the limited support children have in the reading process on the part of their families, which results in a minimal development of reading abilities, in the limited understanding of texts and low academic performance.
\end{abstract}

Key words: Reading comprehension, primary education, family, linguistic abilities

\footnotetext{
${ }_{1}^{1}$ Profesora e investigadora de la Universidad de Costa Rica, Costa Rica. Dirección electrónica: maria.gonzalezaraya@ucr.ac.cr ORCID https://orcid.org/0000-0003$\underline{0626-9660}$

2 Profesora e investigadora de la Universidad de Costa Rica, Costa Rica. Dirección electrónica: helvetia.cardenas@ucr.ac.cr ORCID https://orcid.org/0000-0002-7193$\underline{2900}$
}

Artíuclo recibido: 18 de julio, 2019

Enviado a corrección: 25 de setiembre, 2019

Aprobado: 2 de diciembre, 2019 


\section{Introducción}

Este artículo emerge de la investigación "Los procesos de comprensión lectora y su interacción con las TIC, otros mediadores culturales y la acción pedagógica docente", en el cual se abordó el proceso de la comprensión lectora de la niñez, así como la relación de algunos mediadores culturales como: la familia, las TIC, los recursos didácticos, la mediación pedagógica y el enfoque que utiliza el docente para promocionar la lectura en el aula. El propósito fue determinar el papel y las implicaciones de estos mediadores culturales en el proceso lector de la niñez en algunos grupos de los niveles de Transición y I y II ciclos de la Educación General Básica en la región de Occidente.

Para efecto del presente escrito solamente se retomará la participación de la familia en el proceso de aprendizaje de la comprensión lectora, pues, como mediador cultural, juega un papel muy importante en dicho proceso, puesto que el grupo familiar, junto con los y las docentes y el centro educativo constituyen una red de apoyo importante para que los procesos de aprendizaje sean exitosos. Dada la importancia que ello reviste, se abordó el tema con el alumnado del nivel de Educación Primaria en una escuela de la Dirección Regional de Educación de San Ramón, Alajuela y dos de Grecia, pertenecientes a la Dirección Regional de Educación de Alajuela, Costa Rica.

Al respecto, cabe señalar como resultado que un alto porcentaje de los padres y madres no consideran importante acompañar a sus hijas e hijos en el proceso educativo, lo cual recarga la labor a las personas docentes y diezma en forma significativa la adquisición y desarrollo de habilidades lingüísticas como en este caso, la comprensión lectora, en el ámbito familiar.

Teniendo presente el poco involucramiento o la ausencia de la familia en los procesos de aprendizaje de sus hijas e hijos, este artículo pretende explorar el acompañamiento más intencionado y cercano por parte de los padres y las madres y visualizar el beneficio que ello supondría para la niñez en edad escolar.

\section{Marco teórico}

\subsection{La comprensión lectora y su importancia}

De acuerdo con nuestra experiencia en anteriores investigaciones pudimos observar cómo algunos docentes conceptualizan la habilidad de leer como el simple proceso de decodificación de las palabras por parte de los usuarios. No obstante, leer es un proceso complejo, que requiere del desarrollo de diversas habilidades, pero debido a la poca 
información que se tiene y a una pobre conceptualización de lo que realmente significa, esta es una labor que se le ha delegado a la escuela únicamente y esta, a su vez, lo asume como una tarea que se le designó desde su origen. Quizá de ahí deviene el poco interés que ha mostrado la familia en el proceso de alfabetización inicial y específicamente en el aprendizaje de la lectura, ya que su papel en dichos procesos, con frecuencia, no ha sido relevante y se ha conceptualizado como una obligación del sistema educativo, no así, de la familia.

Por su parte, la familia, asume que sus hijos e hijas leen en tanto, pueden decodificar notas, carteles, rótulos, mensajes e incluso libros completos, sin embargo, como lo señala Cassany, Luna y Sanz, (2005, pp. 194-195), la niñez lo que hace es "discriminar la forma de las letras, establecer la correspondencia entre sonidos y grafías, leer palabra por palabra, pronunciar las palabras correctamente, entender todas las palabras de cada texto", mientras que las otras habilidades se dejan de lado. Desde ahí se da por un hecho, equivocadamente, que ellos adquirieron la habilidad lectora y su comprensión.

Solé (2009) complementa esta afirmación de Cassany cuando señala que "leer es comprender, y comprender es ante todo un proceso de construcción de significados acerca del texto que pretendemos comprender. Es un proceso que implica activamente al lector, en la medida en que la comprensión que realiza no es una derivada de la recitación del contenido de que se trata" (p.37). Por otra parte, para Colomer, "leer es un acto interpretativo que consiste en saber guiar una serie de razonamientos hacia la construcción de una interpretación del mensaje escrito, a partir de la información que proporciona el texto como de los conocimientos del lector" (1997, p. 9).

Cabe agregar que de acuerdo con autores antes citados y los hallazgos de anteriores investigaciones conceptualizamos, además, la comprensión lectora desde un enfoque comunicativo de la lengua como un acto complejo que implica la interacción textointerlocutor. Es decir, que como lectores nos enfrentamos a un texto desde nuestros intereses, gustos y necesidades de información, partiendo de los propios conocimientos y formas de aprehenderlo y para ello utilizamos diversas estrategias metacognitivas que garanticen, en buena medida, relacionar los aprendizajes previos a fin de lograr aprendizajes nuevos y significativos como un constante aprender, reaprender y de desarrollar el análisis y la crítica a la hora de leer. Ello favorece lectores autónomos y críticos en la medida en que afloran nuevas habilidades y destrezas a fin de lograr una determinada finalidad.

Posicionándonos desde un enfoque comunicativo, leer es sobre todo, una acción que conlleva la construcción de significado mediante la información que proporciona el texto 
escrito. Así, más que un acto mecánico de descifrado de signos gráficos, es un acto de construcción personal y subjetivo en donde el lector le atribuye significado al contenido del texto, pues como lo señala Novak y Gowin (1988, p. 65) "la lectura es un medio de aprender significados, pero resulta difícil leer palabras y frases cuando no tienen ningún significado". Es decir, cuando se está comprendiendo, se está incorporando nueva información, elaborando y reelaborando nuevos conocimientos a los esquemas existentes y en diferentes contextos. En este sentido y con base en nuestra experiencia consideramos que la competencia comunicativa promueve el uso de la lengua en forma adecuada y eficaz y nos permite enriquecer la capacidad de comprender, expresarnos y comunicarnos en los diferentes contextos de la vida, pues el conocimiento formal de la lengua facilita la adquisición de la competencia comunicativa, pero no nos habilita para ejercerla asertivamente.

Para Gómez Palacios (1996, p. 49) "la lectura es un proceso interactivo entre el pensamiento, el lenguaje y la comprensión, es la construcción del significado, ya que este no es una propiedad del texto, sino que lo construye el lector, al otorgarle sentido". Siguiendo a esta misma autora, ella señala que "la lectura es un proceso que requiere de la aprehensión del texto, del descubrimiento de los aspectos implícitos en él, lo cual convergerá en la interpretación, formulación de juicios y en la creación de ideas propias en conjunto con aquellas que ofrece el texto".

La lectura es una competencia transversal en el proceso de aprendizaje del estudiantado, la cual se relaciona con el dominio de saberes específicos y el desarrollo de conocimientos creativos, lúdicos y críticos. A este contexto responden las pruebas PISA de las cuales Costa Rica ha sido objeto, aunque no con resultados halagadores, pues, dicha prueba reveló que la tasa de alfabetización es de un $96 \%$ en el país, pero apenas un 33\% del estudiantado obtuvo una comprensión de lectura superior al mínimo. Al respecto señala la Dra. Snow en entrevista dada a La Nación del 18 de marzo del 2013,

La alfabetización del siglo XXI ya no equivale a identificar letras y decodificar palabras; la economía de la información exige personas capaces de analizar, interpretar y crear. El nuevo alfabetismo obliga al sistema educativo y a los niños a pensar y a preguntar desde su temprana infancia. Para lograrlo, debemos ampliar sus bases de información, para que puedan construir a partir de ahí (parr.1) y continúa diciendo que "los retos para leer en una economía de la información van mucho más allá de lo que las escuelas han estado enseñando y parte del problema es que establecimos estándares 
de alfabetismo hace 80 años, época en la que si podías leer un menú o los titulares de un periódico, eras considerado alfabeta".

Al respecto, es posible inferir que en la sociedad de hoy se han establecido exigencias que no existían hace décadas, no sólo de orden teórico, sino también práctico y así lo señala (Chartier, 2000), cuando se refiere a la lectura como un proceso de

desentrañar los sentidos no explícitos de las palabras, juzgar la veracidad de las fuentes, adecuar los registros de habla y de escritura a las circunstancias y los destinatarios, entre otros. Sostener una educación que comunique estas prácticas implica, además, revisar las metodologías tradicionales arraigadas en los sistemas educativos, creadas con fines y contenidos muy diferentes.

Desde esta perspectiva, el Ministerio de Educación Pública como ente rector de la educación costarricense, presentó en abril del 2013 el nuevo Programa de Español para el I ciclo, el cual amplía de uno a dos años el tiempo que se le da a los alumnos para aprehender el proceso de la lectoescritura, pues pretende "integrar el primero y segundo grado de la educación primaria como una sola unidad o proceso de aprendizaje y evaluación para dicho proceso de lectoescritura pueda ajustarse al avance y ritmo de aprendizaje de cada estudiante" (2013, p. 9). Además, señala dicho programa que

parte de un conjunto de reformas curriculares que hemos impulsado en los últimos años, las cuales buscan que los estudiantes aprendan lo que es relevante y que lo aprendan bien: que los jóvenes adquieran y desarrollen el conocimiento, la sensibilidad y las competencias científicas; lógicas y matemáticas; históricas y sociales y, algo especialmente importante para la vida en el mundo de hoy, que desarrollen los conocimientos y las competencias de la comunicación y lenguaje en su sentido más amplio, indispensable para la vida y la convivencia. (p.5)

Este cambio se fundamenta, según lo señala el nuevo programa porque nuestros estudiantes no saben leer y escribir bien. Lo vemos en las pruebas diagnósticas de sexto y noveno año; asimismo, cuando llegan a las universidades y lo vemos en los resultados de las pruebas PISA: apenas una quinta parte de nuestros estudiantes de sexto año poseen las habilidades lingüísticas esperadas para su nivel; el $67 \%$ de nuestros jóvenes posee apenas un dominio básico de lectura y, además, 
muestran dificultades para responder preguntas que requieran mayor análisis e interpretación. (p.6)

Como se observa, la comprensión lectora es una debilidad en nuestro sistema educativo y requiere de una urgente atención por parte del Ministerio de Educación, así como de un esfuerzo significativo para que docentes, padres y madres de familia y el estudiantado lo conceptualice de una manera diferente desde la importancia que reviste, no como simple decodificación, sino como una forma de elaborar los significados, posibilitar la interpretación y el análisis como una habilidad para la vida y así lo señala Gómez, 2000 (citado por Murillo, 2011) "se trata, por ejemplo de aprender a justificar un hecho histórico, de argumentar posibles interpretaciones de un experimento de laboratorio, de comentar una obra artística, de defender una idea, un proyecto, etc." (p.24).

En relación con lo anterior, los hallazgos del Sétimo Informe del Estado de la Educación muestran que "la probabilidad de que los docentes empleen las prácticas de enseñanza de lectura y escritura según la reforma curricular en Español, es, en promedio, de apenas un 50\%".

De acuerdo con lo anterior, se debe tener claridad de que aprender a leer es una destreza estrechamente ligada a la comprensión. De ahí que, a las niñas y los niños que no la hayan desarrollado les resulta muy difícil el aprendizaje de las otras materias, dado que la lengua es el eje del currículo y sin comprensión no se construye un conocimiento adecuado, ni un desempeño aceptable, lo cual conlleva con frecuencia al fracaso escolar. Leer no es decodificar el texto, sino como lo destaca Puente (2001, p. 21) "la lectura, según las concepciones cognitivas, es un proceso de pensamiento, de soluciones de problemas en el que están involucrados conocimientos previos, hipótesis, anticipaciones y estrategias para interpretar ideas implícitas". Es decir, si la comprensión lectora es un proceso complejo, con más razón los padres y las madres deberían acompañar a sus hijos en el proceso de su aprendizaje y en el desarrollo de habilidades con dicho fin.

\subsection{La familia como mediador cultural}

De acuerdo con nuestra labor docente y la experiencia en la investigación que sustenta este artículo consideramos que la familia como mediador cultural reviste una gran importancia, puesto que como institución social, determina la vida del ser humano y ha ejercido gran injerencia a través de la historia en la sociedad en general. Cada familia se 
constituye en un grupo único, en tanto va entretejiendo su propia cultura mediante la construcción de valores y actitudes coherentes con una determinada cosmovisión. De esta manera, regula el accionar de cada uno de los integrantes con mecanismos de aprobación cuando se obedecen los valores aceptados dentro del grupo familiar o de desaprobación, cuando estos se contradicen.

Esta pedagogía familiar se ve concretizada en la interacción de un sistema complejo de intersubjetividades que conforman un accionar unificado y congruente con el proceso de construcción del conocimiento llevado a cabo dentro del seno familiar y cobra relevancia, de acuerdo con los intereses y objetivos que sus miembros establezcan y pretendan lograr.

Esto significa que desde la familia se forma la actitud y la vocación de cada uno de sus integrantes hacia determinadas actividades significativas y procesos de vida y uno de los más importantes, es el educativo. De ahí se deriva la enorme responsabilidad que tienen las familias en el proceso de aprendizaje de sus hijas e hijos, razón por la cual su identificación, compromiso y acompañamiento en todos procesos de la vida, deben ser efectivos para que sean exitosos.

Así lo señalan Funkhouse y Gonzáles 1997:

Ellos obtienen mejores grados y calificaciones más altas en las pruebas, asisten a la escuela con mayor regularidad, cumplen más con sus tareas, demuestran mejor actitud y comportamiento, se gradúan con mayor frecuencia de la escuela secundaria, y tienen mayor tendencia a matricularse en la universidad, que aquellos estudiantes con familias menos involucradas. (p. 1)

Es decir, que el docente o la docente y la institución educativa no satisfacen por sí mismos las necesidades de aprendizaje de los niños y las niñas, sino que las familias deben ser un complemento y entender que la educación de la niñez es una labor compartida. El estudiantado cuando cuenta con la participación familiar se responsabiliza de su quehacer educativo con seguridad en sí mismo y más motivado para lograr los objetivos de aprendizaje que su proceso educativo demanda porque, sienten el reconocimiento de sus familias y que vale la pena esforzarse para el éxito escolar.

Cuando las familias se involucran en los procesos de comprensión lectora de las niñas y los niños poco a poco se proyecta un impacto significativo en el desarrollo de su lenguaje, especialmente cuando se les empieza a leer en forma temprana, pues las palabras despiertan en la niñez la curiosidad por saber su significado, lo cual deviene en el desarrollo 
léxico y aunado a ello, se advierten otras ventajas y fortalezas, las cuales se enumeran a continuación:

- Se desarrolla la expresión oral, cuando se le solicita a las niñas y los niños que repitan lo leído con sus propias palabras o que comenten el contenido del texto. Esto estimula además, la creatividad y la imaginación, dado que cuando un adulto les lee, ellos (as) dejan volar su imaginación y entran en un mundo fantástico, en tanto se identifican con los escenarios y personajes que el texto evoca o que el lector les describe y les ayuda a imaginar.

- Son personas con muchas posibilidades de convertirse en lectores autónomos y muy críticos, puesto que desarrollan competencias lectoras para identificar, con la ayuda del adulto, la intención del texto y las situaciones presentes en este, para discriminar luego, si son buenas o malas. A su vez, el hecho de leer e interpretar los textos en familia les ayuda a construir valores estéticos acerca de los beneficios de la lectura en general, puesto que el adulto le inculca el valor y la importancia del conocimiento que conlleva la lectura y asimismo, les ayuda a interpretar y reflexionar acerca del texto leído.

- Se fortalecen los lazos afectivos, pues de manera conjunta se conforman espacios dialógicos entre padres e hijos cuando leen juntos, ya que se fomenta la discusión y con ello la construcción del conocimiento al compartir las ideas y desarrollar criterios.

- Se contextualizan diferentes situaciones de aprendizaje, ya que mediante el texto se ejemplifica una serie de situaciones de la vida cotidiana. El beneficio se centra en la posibilidad de que el niño o la niña pueda aprender mediante los ejemplos que le muestra el texto.

- Se fortalece la seguridad en la niñez, pues el acompañamiento de su familia en el proceso lector les ayuda a lograr mayor comprensión de los textos y a crecer seguros de sí mismos e independientes.

- Se favorece la memoria, mediante ejercicios mnemotécnicos con los personajes, escenarios, situaciones que el texto ofrece y se promueve la fantasía, al momento que se recrean las lecturas, especialmente cuando son cuentos, leyendas o historias de otros mundos.

- Se fortalece la sana convivencia familiar y la creación de espacios dialógicos donde los padres comparten sus saberes con los hijos, construyen valores a partir del texto o discuten las consecuencias de los malos actos de algún personaje. 
- Se forman niñas y niños con mayores recursos y con un capital cultural que favorece el establecimiento de inferencias y la capacidad de análisis de situaciones de aprendizaje relacionados con situaciones, hechos históricos, personajes, entre otros.

- Se construyen valores estéticos, como el valor del texto, el gusto por la lectura y una verdadera concienciación de su importancia, dado que, a mayores espacios de lectura, mayor aprendizaje y comprensión de la vida.

- Se construyen valores mediante la discusión y análisis de situaciones y conflictos que contienen los cuentos, las historias, las leyendas, las fábulas y parábolas, entre otras especies narrativas.

Por otra parte, El Centro de Documentación e Investigación de Literatura Infantil y Juvenil, de Salamanca, en su libro Familia y lectura (2007), manifiesta que en la sociedad actual, llamada de la información, donde la tecnología ha irrumpido con fuerza, instaurando nuevos modos de comunicación y de construcción de conocimientos, que llenan los espacios virtuales con una cantidad ingente de documentos, educar en la lectura es más difícil que antes, pero también más importante y necesario. (p. 8). En este sentido, cabe destacar que la familia tiene más recursos ahora para iniciar los procesos de lectura en forma temprana, pero también deberá ser cuidadosa de seleccionar adecuadamente los textos que leen sus hijos e hijas, dado que en la red se pone a disposición lecturas de todo tipo.

Aquí cobra validez lo que continúan señalando en dicho centro acerca de que La familia, tiene un papel y una responsabilidad irreemplazables en el desarrollo de los hábitos lectores del niño, a los que podrá contribuir poniéndolo en contacto con buenas y variadas lecturas, próximas a su mundo afectivo y a sus intereses; lecturas que le hagan pensar y que respondan a sus inquietudes, con una variedad de estilos y contenidos que favorezcan la evolución de sus gustos personales y le ayuden a perfilar y recorrer su propio itinerario lector. (p.10), pues según ellos, la familia, sobre todo en las primeras etapas de la vida, constituye una fuente constante de experiencias y aprendizajes. La lectura, durante toda la vida,también. El espacio familiares un contexto idóneo para inculcar valores sociales y hábitos culturales, $\mathrm{y}$ ofrece posibilidades inigualables para contribuir al proceso de crear lectores. (p. 13) 


\section{Método}

\subsection{Enfoque}

Se trató de una investigación cualitativa aplicada, según Vargas (2009), el estudio aplicado establece relaciones teóricas y empíricas con una o varias situaciones específicas. El interés principal es confrontar lo que se propone en las teorías con lo que acontece en la práctica; sigue una lógica de investigación inductiva, porque las situaciones concretas realimentan las valoraciones teóricas.

El diseño de la investigación corresponde a un estudio de casos, con un alcance interpretativo, caracterizado este tipo de investigación por enfatizar en situaciones reales que están sucediendo. Según Bisquera (2009) "Desde esta perspectiva interpretativa se priorizan unos conocimientos relacionados con problemas reales que afectan de manera directa a los receptores de la investigación" (p.25). En el caso particular de la investigación se atiende la experiencia que viven las docentes de tres instituciones educativas con su grupo de estudiantes en relación con el tema de la comprensión lectora, el uso de las TIC y el acompañamiento de las familias. Fortalece el abordaje de problemas cotidianos para profundizar en estos y proponer tanto modos de conceptualización como soluciones. Asimismo, Ramírez (2004) señala que "la investigación aplicada permite de forma constructiva comprender, reflexionar y enriquecer la experiencia, así como a la población participante".

\subsection{Unidades de análisis. Población a la cual se dirigió la investigación.}

Hernández, Fernández, Baptista (2007) afirman que lo primero que debe hacerse para definir la población objeto de estudio es establecer la unidad de análisis (personas, instituciones, organizaciones). Para efectos de esta investigación, la selección de la población participante en el 2017 se realizó de acuerdo con el objetivo planteado, a saber: determinar el papel y las implicaciones de la familia como mediador cultural en el proceso lector de la niñez en los niveles de IV y VI de la Educación Primaria en una escuela de la Dirección Regional de Educación de San Ramón, Alajuela y dos de Grecia, pertenecientes a la Dirección Regional de Educación de Alajuela, Costa Rica y se estableció como unidad de análisis una muestra homogénea. Los participantes fueron estudiantes de ambos sexos, de edades entre los 10 y 12 años, en total 140 estudiantes, así como las familias de la niñez involucrada en la investigación. 
Para iniciar con el proceso investigativo se procedió con una carta en la cual se solicitaba la autorización y los permisos necesarios al Ministerio de Educación Pública. Ello permitiría el ingreso a las escuelas con el fin de realizar las visitas, las observaciones, la autorización para usar medios de videograbación donde se incluyera a los participantes, las entrevistas a las docentes y a la población estudiantil, así como la aplicación de instrumentos de diagnóstico a la población estudiantil durante el ciclo lectivo del 2017, con ello se conocería el nivel de comprensión y prácticas de lectura en el aula y para ello se utilizó el consentimiento informado.

3.2.1 Selección de la muestra o participantes. Para la investigación de la cual se deriva este artículo

Se seleccionaron tres centros educativos como se mencionó con anterioridad y a conveniencia del equipo de investigadoras, considerando como criterio que el

cuarto grado es el año en que comienza el II ciclo y el sexto grado se culmina con la formación recibida durante la Educación Primaria. Además, este último año es el previo al inicio de la educación secundaria, la cual tendrá nuevas exigencias para la preparación estudiantil en relación con los procesos de lectoescritura.

\subsubsection{Nivel educativo de las personas relacionadas con el proyecto}

Se realizó una reunión con los padres y madres de familia de los grupos participantes en la investigación y setenta familias decidieron participar respondiendo un cuestionario, cabe destacar que el interés de las supra citadas familias coincidió con el interés de sus hijas e hijos por participar en la investigación. Conviene indicar que el nivel académico de las familias oscila entre bajo y muy bajo.

\subsection{Técnicas de recolección}

Se emplearon técnicas e instrumentos cualitativos entre ellos: la observación, el cuestionario dirigido a las familias participantes, y el instrumento de diagnóstico para abordar los niveles de comprensión lectora. En el cuestionario se preguntó acerca de varios temas como: la tenencia de libros en la casa, qué tipo de libros, cuáles preferían leer los niños y las niñas, si dedicaban tiempo a la lectura en familia, si asistían a eventos relacionados con la lectura, entre otros. Por otra parte, para realizar el diagnóstico se utilizó una serie de lecturas, las cuales tenían un cuestionario incorporado acerca de su contenido, con preguntas, tanto del nivel literal como inferencial. 
Los instrumentos para la recolección de datos incluyen guías de observación, el cuestionario dirigido a las familias y el diagnóstico para la población estudiantil respectivamente.

La etapa inicial correspondió a la recolección de los datos a través de la aplicación de los cuestionarios. Así mismo se procedió a sistematizar la información de los cuestionarios, y de la observación participante.

Para efectos del presente artículo se trabajó con la categoría las familias y la subcategoría: adquisición de la comprensión lectora de las niñas y los niños en Educación Primaria

De acuerdo con Gibbs (2012), el análisis de los datos permite alguna clase de transformación, es decir, se inicia con gran cantidad de datos cualitativos y "los procesa mediante procedimientos analíticos que dan lugar a un análisis claro, comprensible, penetrante, fiable e incluso original" (pp. 19 - 20). En ese proceso es necesario asegurarnos de que los datos estén completos y tengan la calidad requerida para ser analizados.

Con el fin de determinar la validez de la información recopilada se utilizó el principio de la triangulación múltiple de las fuentes de información, al respecto Cerda (1991) afirma que este procedimiento de análisis utiliza diversas fuentes y métodos de investigación para estudiar un problema. La triangulación se lleva a cabo mediante los datos obtenidos en las observaciones, el cuestionario dirigido a las familias, así como las pruebas del nivel de comprensión de lectura y el análisis de documentos para determinar cómo incide la familia como mediador cultural en el desarrollo y motivación de la comprensión y práctica de la lectura del grupo de estudiantes.

\subsection{Procesamiento de análisis de los datos}

En relación con el análisis de la información, es importante aclarar que para efectos de este artículo solo se analizaron los cuestionarios aplicados a las familias, donde interesaba definir su acompañamiento en el proceso de comprensión lectora, en el tipo de textos que leen las niñas y los niños, la cantidad de lectura de un texto asignado, la asistencia a eventos de lectura en familia con las niñas y niños y la utilización de la biblioteca por parte de las niñas y los niños. Para su análisis se procedió a organizar la información en figuras de manera que se evidenciara las diferentes subcategorías citadas. En relación con la caracterización de la niñez se procedió a realizar un análisis descriptivo de la información de los cuestionarios por medio de gráficos. También resulta conveniente indicar que para el 
caso de la investigación se contó con el consentimiento informado, así como el compromiso de confidencialidad de las personas participantes.

La triangulación buscó relacionar la teoría fundada con la información suministrada por las participantes y aportar confiabilidad al estudio.

\section{Resultados}

A continuación, se muestran algunos resultados, de acuerdo con el análisis de la categoría: las familias y la subcategoría: adquisición de la comprensión lectora de las niñas y los niños en Educación Primaria, según algunas respuestas que brindaron los padres y madres de familia en los cuestionarios aplicados.

En la figura 1 se puede observar que las familias no acompañan a sus hijas e hijos a leer, sino que las niñas y los niños realizan el proceso de aprendizaje de la comprensión lectora solamente con el acompañamiento docente. No obstante, la participación de los padres y las madres se considera esencial, puesto que podrían aportar mucho conocimiento a las debilidades de comprensión que muestra el estudiantado. Es importante entender que por la experiencia, las vivencias y el nivel educativo, muchas veces superior que tienen los adultos, pueden aclarar las dudas generadas por el texto en relación con el vocabulario, los contenidos, las relaciones o referencias que este establece. De ahí que, el hecho de acompañar a la niñez en los procesos lectores sea tan significativo y determinante, tomando en cuenta que las niñas y los niños con frecuencia no contestan un cuestionario o no resuelven un problema, ya sea matemático o de diversa índole, porque no comprenden lo que se les solicita. La figura anterior muestra la resistencia de las familias para involucrarse en el proceso lector de sus hijas e hijos, aunque cabe señalar al respecto, que algunos padres y madres justifican su ausencia en este proceso con su analfabetismo o la poca habilidad que tienen para leer y con mayor razón para comprender los textos. Ello nos refiere a una situación que viene a agravar el panorama y por ende, a una situación digna de ponerle atención. 
Figura 1

Número de estudiantes que indican que su familia sí lo acompaña en el proceso de aprendizaje de la comprensión lectora, región de Occidente en Costa Rica, período 2016-2017

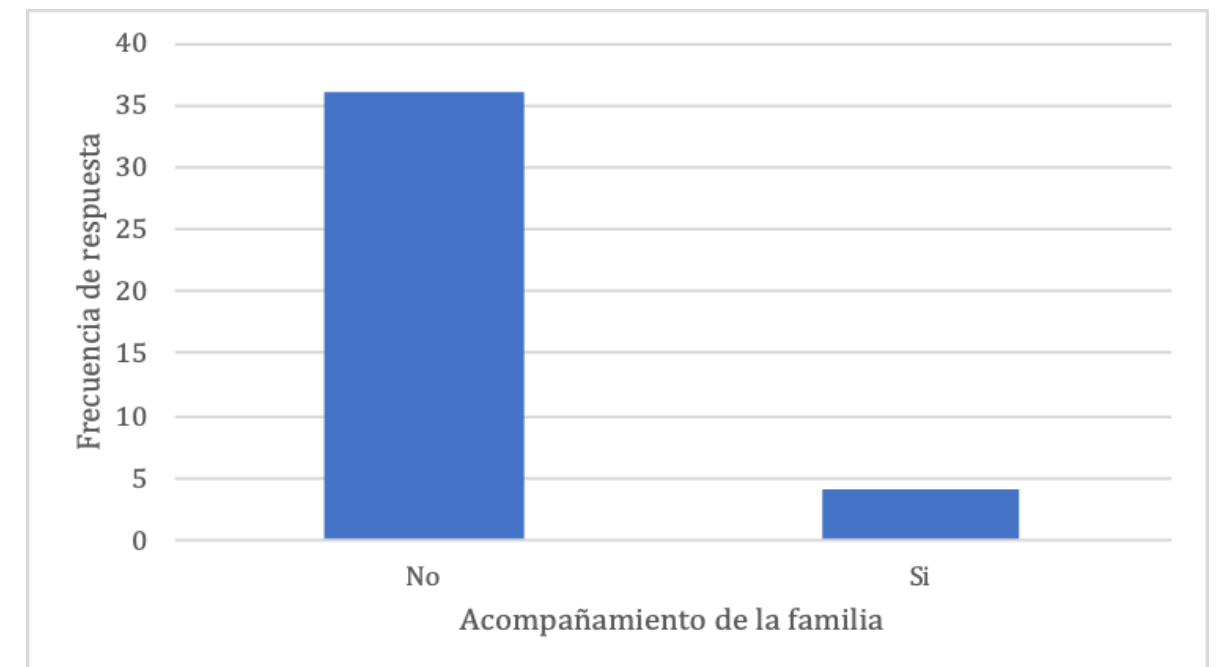

Fuente: elaboración propia con base en las respuestas de los participantes (2018)

En relación con la pregunta que se le realizó a los padres de familia, caracterizados en el apartado de la población participante, acerca de los textos que leen las niñas y los niños o aquellos que prefieren leer, ya sea porque se los asignaron en la escuela o por su propio interés, al respecto contestaron:

Figura 2

Número de estudiantes según el tipo de textos que leen, región de Occidente en Costa Rica, según el Ministerio de Educación Pública, período 2016-2017

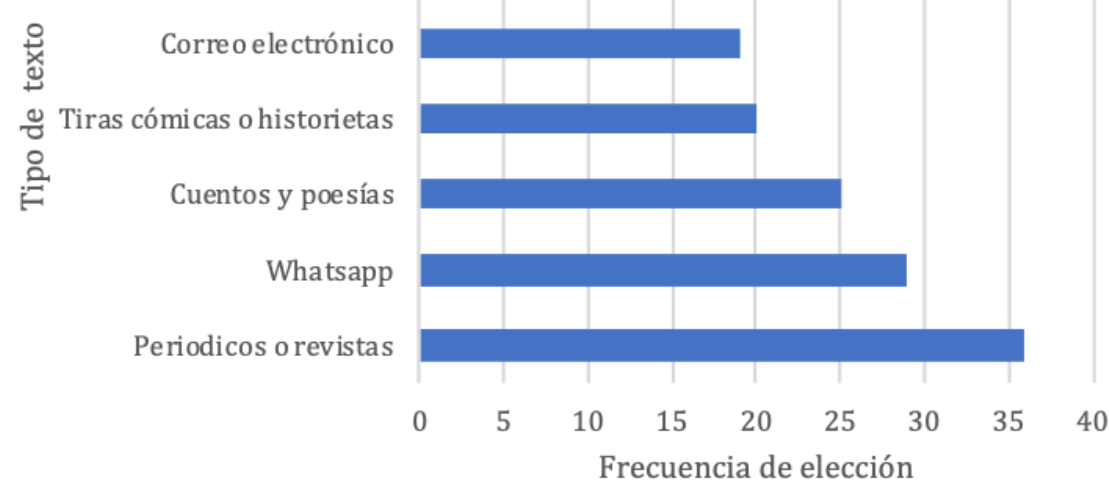

Fuente: elaboración propia con base en las respuestas de los participantes (2018) 
Se puede observar en la figura 2, un significativo interés por parte de la niñez hacia la lectura de periódicos y revistas, así como pequeños mensajes SMS que se transmiten mediante diferentes aplicaciones como es el whatsapp o el correo electrónico. Si bien es cierto, esta mensajería es importante, está en boga y acorde con las nuevas tecnologías, también es cierto que es un tipo de lectura, que por su brevedad, no permite desarrollar habilidades lectoras, puesto que el mismo texto no lo demanda.

La figura 2 nos muestra, además, la importancia que tienen las tiras cómicas 0 historietas para las niñas y los niños, lo cual parece indicar que a ellas y ellos les gusta leer los textos pequeños, sencillos y que no representen mucha dificultad en su comprensión. Se evidencia, también, que la familia al no estar presente en los procesos lectores de las hijas e hijos, dejan en manos de estos últimos la elección del tipo de texto que leen, aunque no sea el más conveniente para su aprendizaje, puesto que no les genera conocimiento alguno. En este sentido, la familia tampoco aboga por la lectura de textos de mayor valor literario, puesto que se mantienen al margen del proceso.

Asimismo, ello es coherente con las respuestas a la pregunta realizada en relación con la extensión o el tiempo de lectura que dedican las niñas y los niños en la escuela o en la casa, ante la solicitud del docente o la docente de leer un libro, un cuento o un texto cualquiera para trabajarlo en clase.

\section{Figura 3}

Número de estudiantes según la cantidad de lectura que lee de un texto asignado, región de Occidente en Costa Rica, período 2016-2017

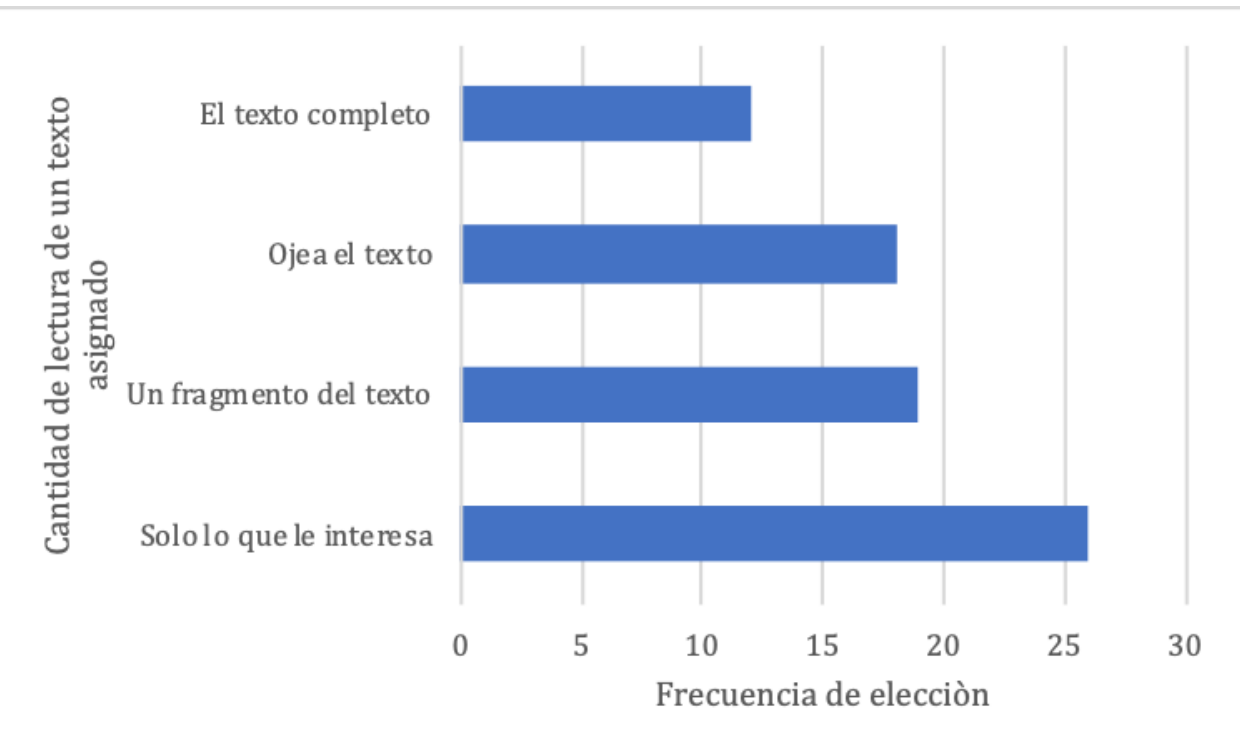

Fuente: elaboración propia con base en las respuestas de los participantes (2018). 
La figura 3 muestra una gran indiferencia ante el texto de corte literario por parte de las niñas y los niños. En opinión de los padres, ellos realizan un esfuerzo para que ellas y ellos se motiven con la lectura de los textos asignados por las y los docentes, pero fueron enfáticos en expresar que a sus hijas e hijos les cuesta trabajo mostrar verdadera motivación y entusiamo por la lectura y que, al respecto, les resulta más atractivo jugar con el teléfono celular que leer un libro. Según ellos, sus hijas e hijos antes de leer un libro, primero lo ojean, observan los dibujos cuando el texto los contiene y por último, se aventuran cuando mucho, a leer un fragmento del texto. Ellos consideran que a las niñas y los niños se les dificulta mucho decidirse a leer porque no es una habilidad que se desarrolle en la escuela y aunado a ello, por la escasa preocupación de los docentes por fomentarla como una habilidad lingüística, al respecto el VII Estado de la Educación señala:

El 74\% de los docentes de primaria ve la lectura como un ejercicio obligatorio, ajeno al gusto y el placer propios de esa experiencia. Es de esperar, por tanto, que en estos casos las acciones y espacios destinados a motivarla \{la comprensión lector\}, sean restringidos, lo se que refleja, por ejemplo en el hecho de que cerca del $26 \%$ de los educadores pone poco o ningún énfasis en prácticas relacionadas con el ámbito de la lectura. (pág. 100)

Cabe resaltar que si la escuela es poco promotora de la lectura, y la familia no brinda el acompañamiento necesario, el desinterés por los espacios de lectura de los niños y las niñas se agudiza, Admiten los padres y las madres, además, que ellos no promueven ni acompañan la lectura en el hogar porque trabajan fuera todo el día, algunos aducen que no saben leer ni escribir o simplemente no les atrae y que hasta el momento no se habían interesado porque sus hijos e hijas leyeran el texto completo que les fue asignado por el o la docente, ya que no consideran importante la lectura en el hogar.

Por otra parte, en relación con la participación de las familias en los eventos de lectura, como ferias de libros, clubes de lectura, concursos de lectura, entre otros, se les preguntó acerca de la frecuencia con la cual asistían ellos junto con sus hijos e hijas a estas actividades y al respecto contestaron: 
Figura 4

Número de estudiantes según asistencia a eventos de lectura, región de Occidente en Costa Rica, período 2016-2017

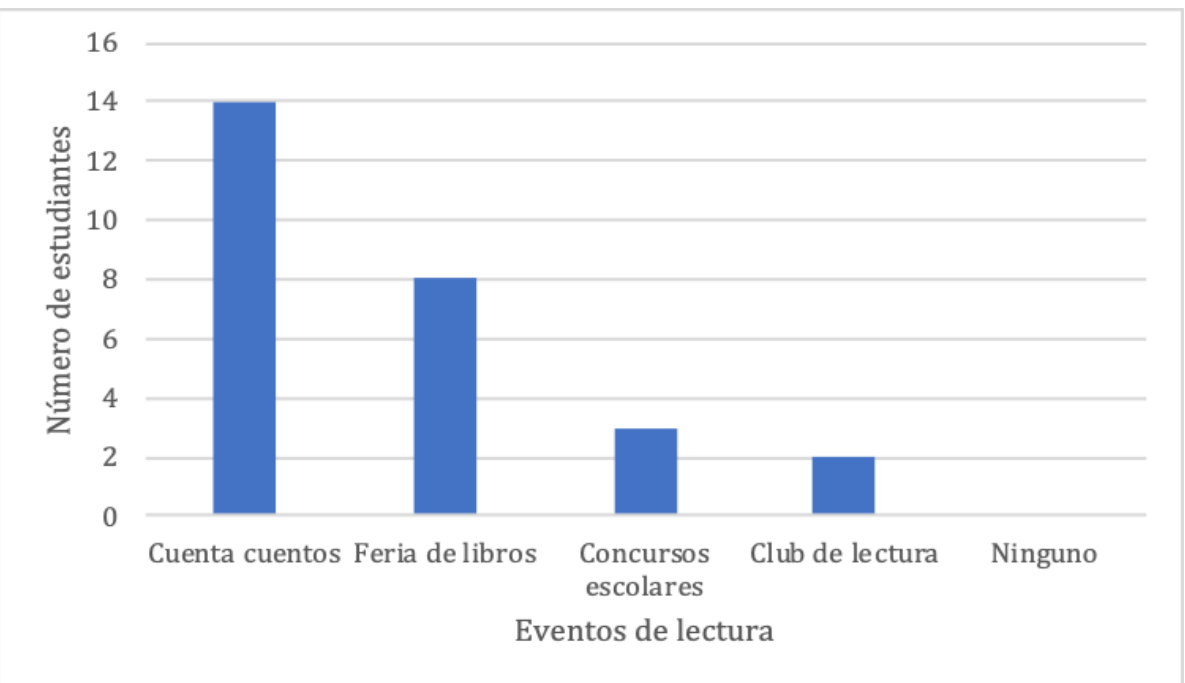

Fuente: elaboración propia con base en las respuestas de los participantes (2018).

Es importante resaltar que si las madres y los padres de familia no asisten a este tipo de eventos, es imposible que las niñas y los niños se motiven en visitarlos o participar en ellos. En la figura 4 se observa una escasa o nula asistencia a este tipo de eventos, lo cual evidencia que la lectura no es un aspecto importante dentro del núcleo familiar, tampoco es un hábito en los padres o las madres de las y los estudiantes y por ende, el gusto por la lectura tampoco se ha desarrollado. Es decir, no es un valor construido desde las familias, quizá porque dentro del seno familiar no se ha reconocido el verdadero potencial que reviste la lectura en la niñez como generadora de conocimiento y de cultura en general. Esta investigación evidenció, además, que en las aulas nuestra niñez lee obligada cuando los docentes así lo solicitan, lo cual representa un sacrificio para una gran mayoría de estudiantes y de igual forma, un reto para el o la docente, puesto que es conocedor del desinterés de la niña o el niño y de antemano sabe que la lectura no significa deleite para el estudiantado. De esta forma, se entra en una especie de círculo vicioso de no asignar lecturas porque al alumno no le gusta o no le interesa, lo cual deviene en un mayor desinterés, poca comprensión y menos conocimiento. 
Figura 5

Número de estudiantes según el tipo de utilidad de la biblioteca, región de Occidente de Costa Rica, periodo 2016-2017

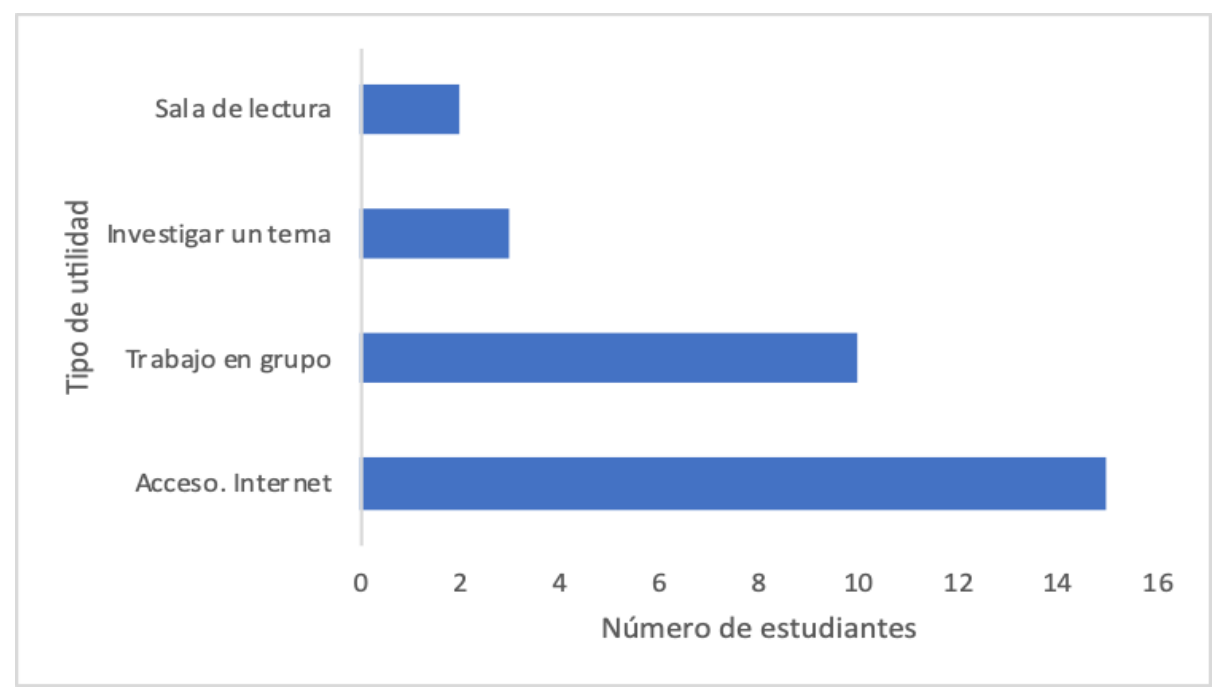

Fuente: elaboración propia con base en las respuestas de los participantes (2018).

De acuerdo con la figura 5, el propósito real de las bibliotecas escolares como espacios de lectura, investigación y de aprendizaje, no se cumple. Al respecto, la presente investigación evidenció que, por una parte, el cuerpo docente no lo considera como un lugar de lectura para el estudiantado, ni siquiera lo procuran como un espacio de consulta de las niñas y los niños cuando les asignan las tareas. Con mucha preocupación se logró observar que las tres escuelas participantes en la investigación conceptualizan la biblioteca como el espacio idóneo para reuniones u otras actividades, distante de su verdadero objetivo. Cabe señalar, que en el caso de las escuelas participantes en la investigación, las bibliotecas son utilizadas mayormente por los estudiantes para acceder a internet, realizar trabajo en grupos, recibir charlas o talleres y en poca medida como espacio de consulta de libros o lectura. Por su parte, la familia tampoco la considera como un lugar de visita frecuente para que la niñez la aproveche leyendo. En su mayoría, los padres y madres justifican su ausencia a estos lugares argumentando que ahora todo se logra por internet, sin embargo, en la mayoría de hogares son escasas las computadoras y carecen de conexión a la red. 


\section{Figura 6}

Número de familias según la cantidad de computadoras por hogar, región de Occidente en Costa Rica, periodo 2016-2017

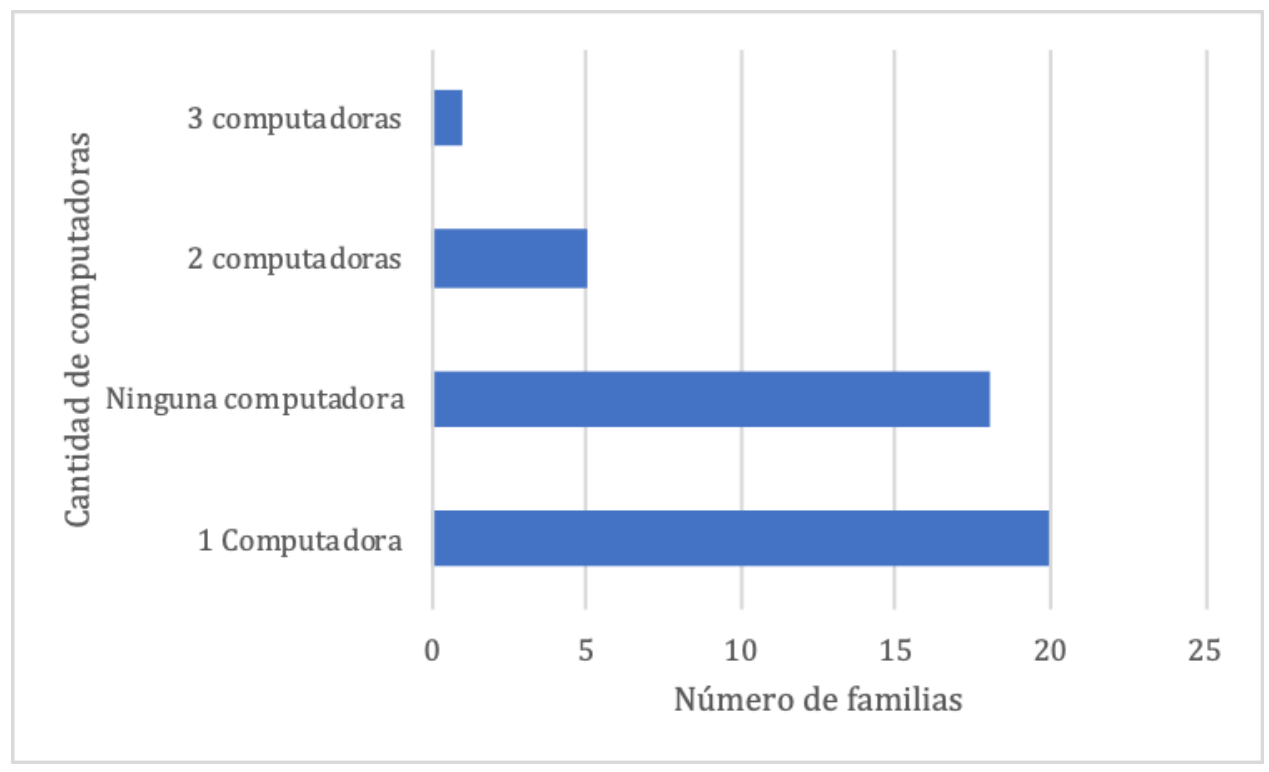

Fuente: elaboración propia con base en las respuestas de los participantes (2018).

De acuerdo con la figura 6 , cabe señalar que las computadoras no son una herramienta de fácil acceso para la niñez de las escuelas estudiadas. Es conveniente anotar que, a pesar de la importancia que reviste el hecho de tener al menos una computadora en el hogar y con todos los beneficios que de ellas podría obtenerse respecto del acceso a la información para complementar los procesos educativos, en la mayoría de los hogares de estos estudiantes no tienen ninguna, lo cual podría interpretarse como una debilidad. De ahí, el interés de las niñas y los niños por utilizar la biblioteca escolar para acceder a internet. Asimismo, es importante destacar que debido al auge de la tecnología, a la niñez le resulta más atractivo el aprendizaje mediante el texto digital que por medio del texto escrito. En este sentido, debe observarse que la escasez de computadoras en los hogares genera el poco acceso a la información por parte de las familias estudiadas, lo cual representa otro aspecto que abona al poco desarrollo del conocimiento y de la habilidad lectora.

\section{Conclusiones}

A continuación se presentan diversas conclusiones, las cuales están relacionadas con los principales aspectos del estudio y en congruencia con el objetivo desarrollado y analizado. 
Desde la experiencia de esta investigación, los docentes reconocen el valor de la familia y su impacto en el rendimiento escolar de los educandos y señalan que esta con frecuencia se ha apartado del proceso de aprendizaje de las habilidades lingüísticas de los niños y las niñas. En este sentido, cabe señalar que la familia debe conformar un todo indivisible con la escuela y una ha de constituirse en el complemento de la otra, puesto que el éxito escolar se promueve también desde el hogar con el debido acompañamiento del padre, la madre y los hermanos mayores hacia el niño en el proceso de aprendizaje. Aunado a ello, esta investigación evidenció que algunos (as) docentes conceptualizan la habilidad de leer como el simple proceso de decodificación de las palabras, lo cual deviene en la poca preocupación por desarrollar habilidades de comprensión lectora con el estudiantado. No obstante, como se ha reiterado, leer es un proceso complejo que requiere del desarrollo de diversas habilidades para que sea un verdadero proceso de aprendizaje y de acuerdo con la Didáctica de la lengua y literatura, la mediación pedagógica de la comprensión lectora, debería orientarse a potenciar lectores autónomos, capaces de interactuar con el texto y de lograr discernimiento y criterio propio, por ello es importante que el docente reconozca la lengua como eje del currículo y no como una asignatura, puesto que la lengua es un vehículo de construcción del conocimiento y mediadora de nuevos aprendizajes, de comprensión y aprehensión de la realidad. Por esta razón,

Cabe destacar, que a pesar de todos los beneficios señalados por el acompañamiento de las familias en el proceso lector de la niñez, este estudio evidenció que, salvo en algunos casos, la familia está prácticamente ausente del proceso de aprendizaje y adquisición de la competencia lectora, lo cual nos llevó a inferir que la niñez participante enfrenta dicho proceso sola y teniendo únicamente al docente como mediador, quien con frecuencia no lee más allá del texto que utiliza en el aula. Por su parte, los padres y madres de familia participantes, en su mayoría, no consideran una prioridad acompañar a sus hijas e hijos en los procesos de comprensión lectora, pues ellos confían su aprendizaje a los y las docentes. A pesar de ello, el cuerpo docente evidenció, en un alto porcentaje, una deficiente aprehensión del referente teórico y conceptual de la Didáctica de la lengua y la literatura, lo cual se proyecta en una pobre mediación pedagógica que deviene en procesos educativos posicionados desde un enfoque gramatical y por ende, un accionar docente distante del enfoque comunicativo, promovido por el Ministerio de Educación en los nuevos programas de Español, publicados en el año 2014 y recomendado, a su vez, por los diferentes autores que se posicionan desde la Didáctica de la lengua y la literatura. Recuérdese que el enfoque 
comunicativo promueve lectores independientes, críticos, analíticos, capaces de hacer de la lengua un instrumento de comunicación en todos los contextos.

En este sentido, la escasa aprehensión de un referente teórico y conceptual de la Didáctica de la lengua por parte del docente, diezma la participación de los padres y madres de familia en el proceso lector de los niños y las niñas, dado que al posesionarse desde un enfoque gramatical, no se les concibe desde su verdadero papel potenciador cuando se trabaja de manera conjunta, docente- alumnado- familia. El o la docente que trabaja desde el enfoque gramatical considera, muchas veces, que leer es simplemente decodificar un texto y para hacerlo el estudiante es autosuficiente, le dan prioridad a la definición de conceptos gramaticales, reconocimiento de oraciones, a la morfología y la sintaxis, pero no así a la comprensión, inferencia e interpretación y análisis de los textos, lo cual genera serias debilidades a nivel de comprensión lectora y nos lleva a inferir que los mismos docentes no han desarrollado la habilidad lectora, por eso no la pueden ejecutar en el aula. Ello es coherente con la satisfacción que sienten al escuchar a la niñez leyendo, pero no comprendiendo.

Es importante señalar que a pesar de que esta investigación se realizó en tres escuelas con modalidades diferentes, a saber, dos rurales y una urbana, el vacío de la familia en el proceso de aprendizaje de la comprensión lectora es muy similar, lo que constituye un dato preocupante, más aún tomando en cuenta que las familias de escuelas urbanas podrían generar mayores expectativas de involucramiento a partir del nivel educativo que poseen los padres, las madres o encargados del estudiantado y del acceso a la tecnología que poseen. Cabe señalar, entonces, que la familia como mediador cultural en los grupos estudiados, no evidenció una injerencia positiva en el proceso lector de la niñez en el nivel de Educación Primaria, lo cual deviene en niveles de comprensión lectora muy elementales y las docentes participantes no evidenciaron la apropiación de un referente teórico y conceptual que les permita, en su accionar, favorecer los procesos de comprensión lectora en el nivel de Primaria, desde un enfoque comunicativo, lo que significa desarrollar con la niñez habilidades lectoras que formen lectores independientes. Por su parte, la familia le ha delegado a la escuela dicha labor y esta lo asume como una tarea que se le designó desde su origen. Quizá de ahí deviene el poco interés que han mostrado las familias en estudio hacia el proceso de la alfabetización inicial y específicamente en el aprendizaje de la comprensión lectora, ya que su papel en dichos procesos, con frecuencia, no ha sido 
relevante y se ha conceptualizado como una obligación del sistema educativo, no de la familia.

Ante esta realidad, cabría preguntarse entonces, que si las niñas y los niños no leen en la escuela, ni la lectura es una prioridad en los centros educativos, los padres y las madres podrían convertirse en una excelente opción para acompañar a sus hijos e hijas en el desarrollo de la competencia lectora desde espacios más cálidos, divertidos, lúdicos y bonitos como es la lectura en el seno familiar. En consecuencia, se revela una necesidad urgente del involucramiento de la familia en el proceso lector de las niñas y los niños en el nivel de Educación Primaria, particularmente en el fortalecimiento y adquisición de habilidades de comprensión lectora como una habilidad para la vida.

\section{Recomendaciones}

De acuerdo con los hallazgos de esta investigación se formulan algunas recomendaciones, las cuales están dirigidas a las instituciones educativas y a las familias, con el afán de que las consideren en beneficio de las niñas y los niños para logren procesos exitosos de aprendizaje de la comprensión lectora.

En primera instancia es imperativo establecer nexos entre la institución educativa y las familias a fin de construir valores estéticos en relación con los beneficios que conlleva la adquisición y el desarrollo de la comprensión lectora en el seno familiar, ello considerando, además, que una familia que lee construye el conocimiento y valores estéticos como el gusto por la lectura y se apropia del capital cultural que le ofrecen los textos.

En consecuencia, se debe promover desde las instituciones educativas la participación familiar en los procesos de aprendizaje, específicamente en el de la comprensión lectora, ya sea con un proyecto de centro educativo donde la lectura se constituya en un eje curricular de trabajo conjunto con los padres de familia y no en un contenido más de la asignatura de Español. Es importante comprender que los retos de las sociedades actuales cada vez son mayores y uno de ellos es que el y la estudiante desarrolle la comprensión e interprete críticamente los textos para que pueda aplicar lo aprendido a situaciones nuevas de la cotidianeidad, asimismo, que valore la lectura como fuente de información y aprendizaje del contexto y reconozca su finalidad comunicativa.

Las familias y las escuelas deben fomentar una cultura lectora para lograr que las nuevas generaciones se formen como lectores para la vida; es decir, que hagan de la lectura una práctica cotidiana, pero para lograrlo no basta con dominar la técnica de la lectura, esta 
debe manifestarse en los diversos espacios y momentos de la vida. En el caso de los padres y madres, se trata de promover una cultura lectora en los hogares, lo cual implica que debe haber libros en las casas y que se realicen prácticas lectoras de manera natural. Un hogar lector tendrá libros de diferentes temáticas para todos los gustos y el hecho de asociar la lectura con el gusto y el placer es una manera de hacerla parte de los tiempos de ocio y conceptualizarla como una oportunidad para que padres e hijos lean juntos y compartan momentos significativos en la vida familiar donde se generen espacios lúdicos de lectura con textos diferentes de los que se leen, por obligación, en las escuelas.

\section{Referencias}

Bisquerra, Rafael. (2009). Metodología de la investigación educativa (2 ${ }^{\mathrm{a}}$ ed). Barcelona, España: Editorial La Muralla.

Casals, Albert; Vilar, Mercé y Ayats, Jaume. (2008). La investigación-acción colaborativa: Reflexiones metodológicas a partir de su aplicación en un proyecto de música y lengua. Revista Electrónica Complutense de Investigación en Educación Musical, 5(4). Recuperado de https://revistas.ucm.es/index.php/RECl/article/view/9555

Cassany Daniel, Luna Marta y Sanz Gloria (2005). Enseñar Lengua. Barcelona, España. Editorial Grao.

Catalá Gloria, Catalá Mireia, Molina, Encarna y Monclús, Rosa. (2001). Evaluación de la comprensión lectora. Barcelona: Editorial GRAÓ.

Centro de Documentación e Investigación de Literatura Infantil y Juvenil. (2007). Familia y lectura. Junta de Castilla y León. Consejería de Cultura y Turismo. Gráficas LOPE. Salamanca. España

Cerda, Hugo. (1991). Los elementos de la investigación. Cómo reconocerlos, diseñarlos y construirlos. Bogotá: Editorial El Búho LTDA.

Colomer, Teresa. (1997). La enseñanza y el aprendizaje de la comprensión lectora. Signos, (20), 6-15.

Chartier, Róger. (2000). Las revoluciones de la cultura escrita. Barcelona: Gedisa.

Funkhouse, J. E. y Gonzales, M. R. (1997). La participación familiar en la educación de los niños: abordamientos locales exitosos. Recuperado de http://www.ed.gov/pubs/Famlnvolve/execsumm.html

Gómez Palacios, Margarita. (1996). El niño y sus primeros años en la escuela. Biblioteca para la actualización del maestro. SEP. México. 
Gómez, Isabel. (2000). Bases teóricas de una propuesta didáctica para favorecer la comunicación en el aula. En Jaume Jorba; Isabel Gómez y Angels Prat (coord.), Hablar $y$ escribir para aprender (pp. 19-28). Barcelona: Universitat Autónoma de Barcelona/Síntesis.

Gibbs, Graham. (2012). El análisis de datos cualitativos en Investigación Cualitativa. Madrid, España: Ediciones Morata.

Hernández Sampieri, Roberto; Fernández Collado, Carlos y Baptista Lucio, Pilar. (2007). Metodología de la investigación (4a. ed.). México: Mc Graw-Hill Interamericana.

Ministerio de Educación Pública. (2013). Programa de Español I ciclo. San José, Costa Rica: El Ministerio.

Novak, Joseph Dónald y Gowin, Bob. (1988). Aprendiendo a Aprender. Barcelona España: Ediciones Martínez Roca. S.A.

Programa Estado de la Nación. (2017). Sexto Informe estado de la educación. San José, C.R: Servicios Gráficos, A.C.

Programa Estado de la Nación (2019). Séptimo Informe estado de la educación. San José, C.R.: Masterlitho.

Puente, Aníbal. 2001. Cómo formar buenos lectores. En Pedro Cerrillo y Jaime García Padrino (coord), Hábitos lectores y animación de la lectura (pp. 21-46). Cuenca: Universidad de Castilla La Mancha.

Rosi, Mauro. (2008). La UNESCO y las políticas nacionales del libro. ¿Hacia un nuevo paradigma? Foro Iberoamericano sobre el libro, la lectura y las bibliotecas en la sociedad del conocimiento. Recuperado de http://www.cerlalc.org/Prospectiva/Politicas Libro Unesco.pdf

Snow, Catherine. (18 de marzo 2013). La alfabetización del siglo XXI ya no equivale a identificar letras y decodificar palabras. La Nación (Costa Rica), p. 8A.

Solé, Isabel. (2009). Estrategias de lectura. Barcelona: Graó.

Vargas, Zoila. (2009). La investigación aplicada: una forma de conocer las realidades con evidencia científica. Revista Educación, Universidad de Costa Rica, 33(1), 155-165. 
Revista indizada en

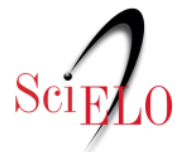

redalyc satindex

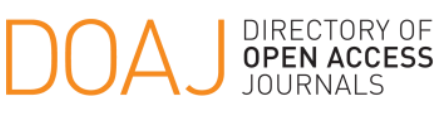

Distribuida en las bases de datos:

- Dialnet

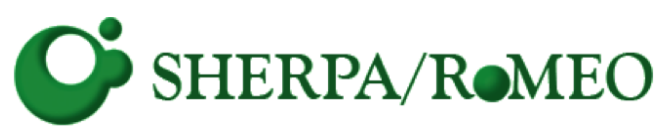

REDIB

Red Iberoamericana

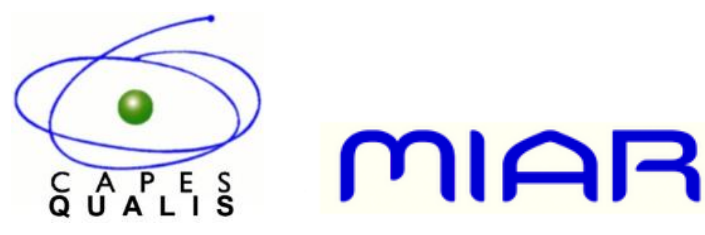

